\title{
Does concomitant meniscectomy affect medium-term outcome of anterior cruciate ligament reconstruction? A preliminary report
}

\author{
Przemysław T. Paradowski ${ }^{1-3}$, Rafał Kęska ${ }^{1}$, Dariusz Witoński ${ }^{1}$
}

${ }^{1}$ Department of Reconstructive Surgery and Arthroscopy of the Knee Joint, Medical University, Radliński Hospital, Lodz, Poland

2Department of Orthopedics, Haugesund Hospital, Helse Fonna, Haugesund, Norway

${ }^{3}$ Department of Orthopedics, Sunderby Central Hospital of Norrbotten, Lulea, Sweden

Submitted: 6 January 2013

Accepted: 16 February 2013

Arch Med Sci 2014; 10, 5: 992-998

DOI: 10.5114/aoms.2014.46219

Copyright $\odot 2014$ Termedia \& Banach

\section{Abstract}

Introduction: Anterior cruciate ligament $(\mathrm{ACL})$ injury is often accompanied by injuries of the menisci. In order to restore knee stability, anterior cruciate ligament reconstruction (ACLR) is performed, with meniscus surgery when needed. The purpose of this study was to assess the medium-term outcome of ACLR in subjects with and without concomitant meniscus tear and partial meniscectomy.

Material and methods: We prospectively studied 73 patients after arthroscopically assisted bone-patellar tendon-bone ACLR. Subjects were divided into two subgroups: those who had only ACLR (group A, 54 subjects with mean age 28, (SD 9)) and those who underwent both ACLR and concomitant partial meniscectomy (group B, 19 subjects, mean age 32 [11]). Subjects completed a disease-specific questionnaire, the Knee injury and Osteoarthritis Outcome Score (KOOS), preoperatively and at a minimum follow-up of 1 year.

Results: No differences in mean scores between group A and B were observed either preoperatively or at follow-up. We found a significant change in mean score in the KOOS subscale QoL in group A following ACLR $(\Delta=$ 9 points, $p=0.039$ ). Most subjects improved in the KOOS subscales Sports and Recreation and QoL in both group A ( 59 and $52 \%$ respectively) and B (63 and $47 \%$ respectively). Eight subjects (15\%) from group $A$ and 1 (5\%) from group $B$ fulfilled criteria of functional recovery. Criteria of treatment failure were fulfilled in 17 subjects (32\%) from A and 4 (21\%) from group B.

Conclusions: Patients undergoing ACLR with partial meniscectomy had a similar medium-term outcome compared to individuals with $\mathrm{ACL}$ tear alone.

Key words: anterior cruciate ligament, outcome, meniscectomy, functional recovery, treatment failure.

\section{Introduction}

The incidence of anterior cruciate ligament $(A C L)$ injuries in the general population has not yet been fully determined but it is estimated at 37$81 / 100,000$ inhabitants per year [1, 2]. Anterior cruciate ligament injury is often associated with concomitant damage of other joint structures, of which injury of the menisci is the most common [2,3]. Due to the altered

\author{
Corresponding author: \\ Przemysław T. Paradowski \\ Department of \\ Reconstructive Surgery \\ and Arthroscopy \\ of the Knee Joint \\ Medical University \\ Radliński Hospital \\ 75 Drewnowska St \\ 91-002 Lodz, Poland \\ Phone: +48 505724726 \\ E-mail: \\ przemyslaw.t.paradowski@ \\ gmail.com
}


biomechanics and ongoing knee instability, the number of meniscus tears in ACL-deficient knees increases over time $[4,5]$.

Since reconstruction of the ruptured $\mathrm{ACL}$ might restore knee stability and reduce the risk of further meniscal and/or cartilage damage, it has widely been accepted as a standard surgical procedure, especially in active young subjects [6]. However, clinical analyses show that one third of subjects undergoing $A C L$ reconstruction (ACLR) have residual instability at follow-up and never reach the pre-injury activity level [7]. Data on whether accompanying knee injuries substantially affect patients' outcome are not consistent. Some authors have found concomitant ligamentous and meniscus injuries not to be significant predictors of patient-oriented outcome [5, 8, 9], whereas others reported worse functional outcome in subjects undergoing ACLR together with meniscus surgery as observed in both medium[10] and long-term follow-up [11, 12]. It has also been proved that additional meniscus surgery can decrease the desired activity level and shorten the career in competitive athletes [13].

We therefore decided to conduct a longitudinal study in patients undergoing ACLR with the focus on identifying factors associated with variability in outcome scores. In this preliminary report we assess the medium-term outcome of ACLR in subjects with and without concomitant meniscus tear and meniscectomy.

\section{Material and methods}

\section{Study sample}

All patients who had undergone ACLR at our department between January 2007 and November 2011 were identified according to surgical records. The major exclusion criteria were good effect of rehabilitation, collateral or posterior cruciate ligament rupture, chondral lesions assigned to chondroplasty, meniscus rupture assigned to suturing and previous knee surgery (Figure 1). We identified 101 patients who completed a self-administered questionnaire evaluating their knee-specific symptoms and knee function. Of them, 73 subjects with a minimum follow-up of 1 year formed the study group. The whole sample consisted of those who had only ACLR (group A) and those who underwent both $A C L R$ and concomitant partial meniscectomy (group B) (Figure 1).

\section{Surgery and rehabilitation}

The postoperative protocol for both groups $A$ and $B$ was identical. All patients underwent standardized arthroscopic single incision bone-patellar tendon-bone autograft ACLR. An additional partial meniscectomy (where no more than half of the meniscus was resected) was performed in subjects with meniscus tears. Patients with meniscus tears that were suitable for suturing were excluded from the study (Figure 1).

All patients had undergone a standardized, moderately accelerated, 6 months' rehabilitation program [14]. Immediate active full extension and flexion up to $90^{\circ}$ of the operated knee was introduced. Free range of motion was permitted 2 weeks after the surgery. The patients were allowed to ambulate 1 day after the surgery and bear weight as tolerated. Crutches were used when needed up to 4 weeks. No knee brace was used. Closed-chain exercises were started gradually postoperatively. Running was permitted three months and contact sports 6 to 9 months postoperatively, provided that the patient had regained

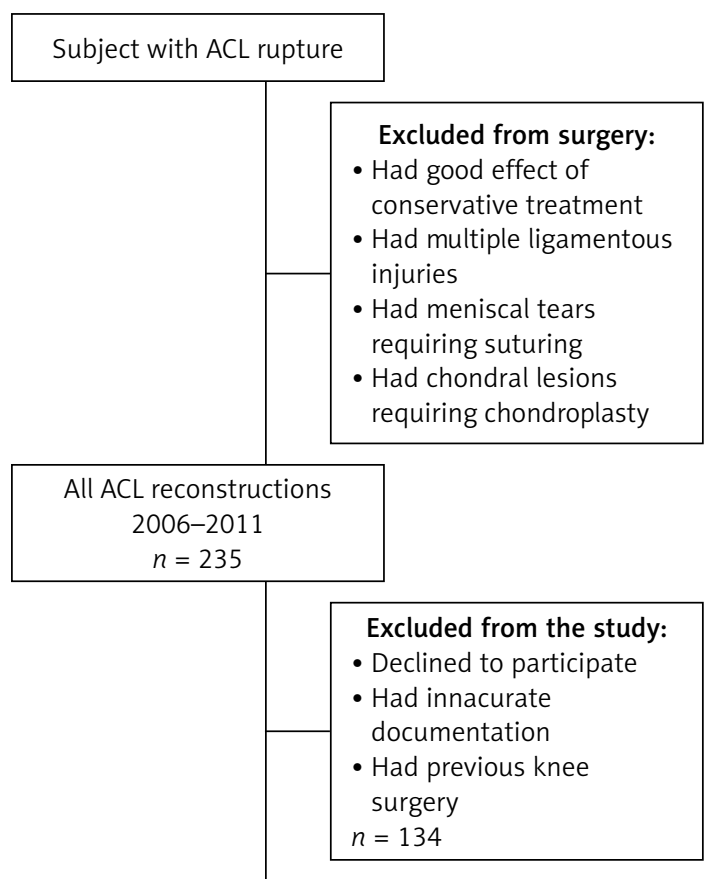

Participants in pre- and postoperative assessment $n=101$
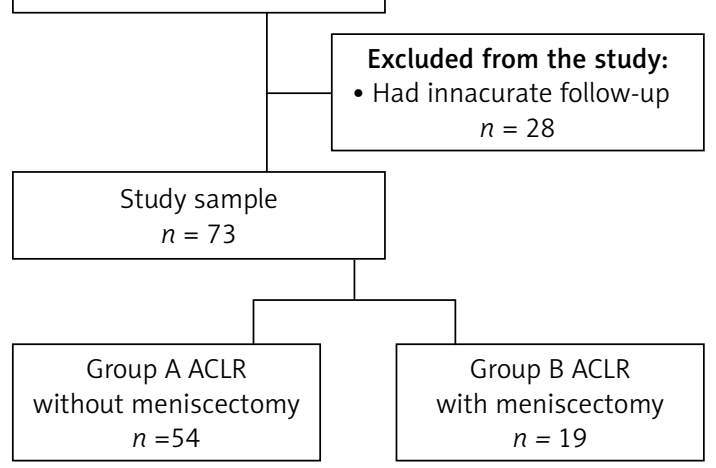

Figure 1. Flow chart presenting inclusion and exclusion criteria for patients. ACL denotes anterior cruciate ligament 
functional stability, muscle strength and coordination.

All the subjects were followed up after they had returned to their normal activities.

\section{Clinical assessment}

The assessment was performed first preoperatively, then during the routine follow-up at a minimum of 1 year after ACLR. All subjects underwent clinical evaluation encompassing the range of motion activity in the operated knee (assessed with a goniometer), wound healing complications, infections, number of aspirations for hemarthrosis and incidence of limb swelling.

\section{Disease-specific questionnaire}

The Knee injury and Osteoarthritis Outcome Score (KOOS) was used. KOOS is a 42-item self-administered knee-specific questionnaire that was developed to be used for short- and long-term follow-up studies of knee injuries and knee OA and is commonly used to evaluate the effect of orthopedic surgery including ACLR [15-17]. The score contains five subscales: Pain, other Symptoms, Activities of Daily Living (ADL), Sports and Recreation and Quality of Life (QoL). A separate score ranging from 0 to 100 where 100 represents the best result is calculated for each subscale. The score had already been validated for ACLR, and culturally adapted for Polish ACL-reconstructed patients [18]. Participants were asked to complete the KOOS questionnaire preoperatively and during the routine follow-up.

\section{Outcome measures}

Since the KOOS subscale ADL was reported not to be sensitive enough to detect changes in patients with ACL injury [16], we defined the primary outcome as a change from baseline to follow-up assessment in the average score for four other KOOS subscales, covering Pain, Symptoms, Sports and Recreation, and QoL (KOOS $)$, with scores ranging from 0 (worst) to 100 (best) $[15,16,19]$. Secondary outcomes included results on all five KOOS subscales and analysis of functional recovery and treatment failure.

\section{Functional recovery and treatment failure}

Based on the published Swedish reference population, functional recovery (FR) level was defined as the lower threshold for the $95 \% \mathrm{Cl}$ of 18-34-year-old males from a reference population [20], as a KOOS score above: 90 for Pain, 84 for Symptoms, 91 for ADL, 80 for Sports and Recreation, and 81 for QoL. Treatment failure (TF) was defined as the KOOS subscale QoL score < 44 [5].

\section{Clinically significant difference}

The minimal perceptible clinical improvement $(\mathrm{MPCl})$ represents the difference on the measurement scale associated with the smallest change in the health status that could be detected by the patient. A level of 10 points or more on a $0-100$ scale was established as a cut-off representing a clinically significant difference [21, 22].

\section{Ethics}

The study was approved by the local ethics committee (approval no. RNN/190/07/KB). Informed written consent was obtained from all subjects participating in the study.

\section{Statistical analysis}

Continuous outcomes are given as mean (standard deviation, SD) values. No prior sample size determination was made due to the observational character of the present study. However, a post hoc power calculation for unequal variances was performed. Statistical power was calculated to be $52 \%$ to detect a $10 \%$ difference of the primary outcome between groups with statistical significance at $\alpha=0.05$. Between-group comparisons of the primary outcome factor were made using general linear model analysis. A confidence interval excluding differences greater than 10 units between groups was interpreted as indicating the absence of a clinically significant difference [17]. We used the Wilcoxon signed ranks test for assessment of comparisons between groups. Binary data in $2 \times 2$ tables were evaluated by Fisher's exact test. Values of $p<0.05$ were considered significant. All analyses were performed with SPSS for Windows 15.0 software package (SPSS Inc., Chicago, IL, USA).

\section{Results}

\section{General characteristics}

The study sample consisted of 54 subjects in group A with a mean age of 28 years (median: 25 , range: $15-54$ years) and 19 subjects in group $B$ with a mean age of 32 years (median: 27, range 18-62 years). No significant differences in age between groups were observed.

No significant differences between age of men and women were observed (mean 27 (SD 7) vs. mean 31 (SD 11) years in group $A$ and mean 31 (SD 9) vs. mean 37 [17] in group $B, p=0.15$ and 0.36 respectively). The mean follow-up time was 1.6 years (range: $1.0-3.0$ ) in group A and 1.8 years (range: 1.1-3.4) in group B. Subject characteristics are given in Table I.

\section{Clinical assessment}

All patients from both groups achieved $90^{\circ}$ of knee flexion on the first postoperative day and full 
extension 2 weeks postoperatively. The full range of motion was gained within 6 weeks postoperatively. No postoperative complications were reported in the study groups.

\section{Patient-relevant outcome}

\section{Score changes on a group level.}

\section{Analysis of the primary outcome factor}

The mean $\mathrm{KOOS}_{4}$ score did not change sufficiently in either group to show improvement at follow-up. No differences in mean $\mathrm{KOOS}_{4}$ scores between group $A$ and $B$ were observed either preoperatively or at follow-up (Table II).

\section{Score changes on a group level. \\ Analysis of the subscale score}

Mean scores changed at follow-up in group A for the KOOS subscale QoL $(\Delta=9$ points, $p=$ 0.039). We observed that mean scores in the subscale Sports and Recreation in subjects from group $A$ and in subscales Sports and Recreation and QoL in group B following ACLR were higher than scores before surgery. However, the increase of these scores did not reach the level of significance $(\Delta=10$ points, $p=0.063, \Delta=13$ points, $p=0.21$ and $\Delta=11$ points, $p=0.25$ respectively). Mean scores for other KOOS subscales did not change significantly following ACLR in both study groups (Table II).

No differences in mean scores between group $A$ and $B$ were observed either preoperatively or at follow-up.

\section{Individual subjects' changes}

We found substantial intra-individual variability when comparing the individual subjects' scores before ACLR and at follow-up. Improvement in $\mathrm{KOOS}_{4}$ was observed in $46 \%$ of subjects from group $A$ and $37 \%$ of subjects from group B (Table III).
Table I. Subject characteristics

\begin{tabular}{|lcc|}
\hline Characteristics & Group A & Group B \\
\hline N \% females & $54(30)$ & $19(26)$ \\
\hline Age, mean (SD) [years]: & & \\
\hline ACLR & $28.1(8.8)$ & $32.3(11.7)$ \\
\hline Follow-up & $29.7(8.7)$ & $34.1(11.7)$ \\
\hline Time to follow-up & $1.6(0.5)$ & $1.8(0.6)$ \\
\hline
\end{tabular}

$N$ - number of subjects, $A C L R$ - anterior cruciate ligament reconstruction

Most subjects improved in the KOOS subscales Sports and Recreation and QoL in both group A (59 and 52\% respectively) and B (63 and 47\% respectively). The percentage of individuals who improved and deteriorated in other KOOS subscales was similar in both groups (Table III).

\section{Functional recovery and treatment failure}

Eight patients (15\%) from group $A$ and one patient from group B (5\%) fulfilled the criteria of FR following ACLR. The number of subjects who scored over the threshold for functional recovery in separate KOOS subscales was as follows: 28 (52\%) for Pain, 27 (50\%) for Symptoms, 34 (63\%) for ADL, 19 (35\%) for Sports and Recreation and 9 (17\%) for QoL in group A and 8 (42\%) for Pain, 6 (32\%) for Symptoms, 11 (58\%) for ADL, 3 (16\%) for Sports and Recreation and 1 (5\%) for QoL in group $\mathrm{B}$.

There were 17 patients (32\%) in group A and four subjects in group B $(21 \%)$ who fulfilled the criteria of TF.

\section{Discussion}

We found that the medium-term clinical outcome in patients undergoing anterior cruciate

Table II. $\mathrm{KOOS}_{4}$ and KOOS subscale scores in subjects from group A (anterior cruciate reconstruction (ACLR) alone, $N=54$ subjects) and B (ACLR and meniscectomy, $N=19$ subjects) before surgery and at follow-up

\begin{tabular}{|lcccc|}
\hline \multirow{2}{*}{ KOOS subscales } & \multicolumn{2}{c}{ Preoperatively } & \multicolumn{2}{c|}{ At follow-up } \\
\cline { 2 - 5 } & Group A & Group B & Group A & Group B \\
\hline KOOS $_{4}$ & $65.8(17.8)$ & $62.3(18.7)$ & $70.8(19.5)$ & $70.5(14.6)$ \\
\hline Pain & $84.5(14.8)$ & $81.1(14.8)$ & $85.5(15.0)$ & $83.1(14.5)$ \\
\hline Symptoms & $79.3(15.6)$ & $70.7(18.3)$ & $79.3(17.4)$ & $78.9(12.7)$ \\
\hline ADL & $87.8(14.7)$ & $88.3(11.2)$ & $89.1(15.3)$ & $90.7(9.8)$ \\
\hline Sports/Rec & $51.7(28.7)$ & $52.9(24.6)$ & $61.7(31.0)$ & $65.5(24.4)$ \\
\hline QoL & $47.8(22.8)$ & $44.6(23.2)$ & $56.5(25.0)^{\star}$ & $55.7(16.6)$ \\
\hline
\end{tabular}

$\mathrm{KOOS}_{4}$ is the change from baseline to follow-up assessment in the average score for four of the five KOOS subscales, covering Pain, Symptoms, Sports and Recreation and QoL. KOOS scores range from 0 to 100, worst to best. Values are presented as means (SD); ${ }^{*} P<0.05$, versus subscale's outcome before ACLR. 
Table III. Number of subjects who reported $\mathrm{KOOS}_{4}$ and KOOS score change at follow-up compared with before surgery. Cut-off for clinically significant difference was set at 10 points

\begin{tabular}{|lcccccc|}
\hline \multirow{2}{*}{$\begin{array}{l}\text { KOOS } \\
\text { subscales }\end{array}$} & \multicolumn{5}{c}{ Group A } & \multicolumn{3}{c|}{ Group B } \\
\cline { 2 - 6 } & \multicolumn{5}{c}{$\boldsymbol{N}(\%)$} & \multicolumn{3}{c|}{$\boldsymbol{N}(\%)$} \\
\cline { 2 - 6 } & Improvement & No change & Deterioration & Improvement & No change & Deterioration \\
\hline KOOS $_{4}$ & $25(46)$ & $14(26)$ & $15(28)$ & $7(37)$ & $7(37)$ & $5(26)$ \\
\hline Pain & $17(31)$ & $25(46)$ & $12(22)$ & $4(21)$ & $11(58)$ & $4(21)$ \\
\hline Symptoms & $17(31)$ & $19(35)$ & $16(30)$ & $7(37)$ & $7(37)$ & $5(26)$ \\
\hline ADL & $13(24)$ & $32(59)$ & $9(17)$ & $3(16)$ & $13(68)$ & $3(16)$ \\
\hline Sports/Rec & $32(59)$ & $6(11)$ & $16(30)$ & $12(63)$ & $2(11)$ & $5(26)$ \\
\hline QoL & $28(52)$ & $10(19)$ & $16(30)$ & $9(47)$ & $5(26)$ & $5(26)$ \\
\hline
\end{tabular}

$\mathrm{KOOS}_{4}$ is the change from baseline to follow-up assessment in the average score for four of the five KOOS subscales, covering Pain, Symptoms, Sports and Recreation and QoL. Values are presented as $N=$ number of subjects and \% of whole group. Cut-off for clinically significant change $=10$ points.

ligament reconstruction (ACLR) alone was not superior to the outcome in patients who had both ACLR and concomitant partial meniscectomy. No significant differences were observed either in the primary outcome $\mathrm{KOOS}_{4}$ or in any of the separate KOOS subscales.

The present study was not the first to investigate the results of ACLR in patients undergoing or not undergoing meniscectomy [3, 10, 11, 23], but to our best knowledge it was the first to find no difference between these groups in medium-term assessment.

Lack of differences in clinical outcomes in patients who had ACLR and those who underwent ACLR together with concomitant knee surgery has been reported to date in one study. This investigation was, however, carried out 5 to 9 years after the surgery and assessed subjects not only with meniscus tears but also with collateral ligament reconstructions [8].

Our results are not consistent with the hypothesis that since the meniscus tear and meniscal resection themselves worsen knee stability, the clinical outcome after ACLR and partial meniscectomy should be worse than after ACLR alone, which, in fact, was observed in several investigations [10, 24, 25].

The ACL rupture is often accompanied by meniscus injury. Several authors have reported that meniscus tears, both medial and lateral, are seen in more than $30 \%$ of knees in their series [26-28]. Many such patients undergo meniscus surgery only and are assigned to rehabilitation due to knee instability. Others are subjected to either early or delayed ACLR. It has been reported that about $14 \%$ of patients undergoing ACLR in Denmark [29] and 20\% in Sweden [5] had surgery of the meniscus prior to ACLR. Subjects with knee operations performed before ACLR were excluded from our study.
Since it has been established that both the functional status and quality of life can be better described by patients themselves than by physicians making clinical examinations, in most ACLR studies the assessment with patient-related outcomes (PROs) is used. However, it has been proved that different PROs have different abilities to capture symptoms and disabilities not only experienced by but also important for patients undergoing ACLR [30]. The inconsistency between our and others' results may thus be due to the method of assessment. Most of the investigators who reported better outcome results of ACLR alone than ACLR with concomitant partial meniscectomy carried out their assessments using the International Knee Documentation Subjective Knee Form (IKDC) [10, 24, 25]. In our study we used the KOOS, a PRO measure which has recently been culturally adapted and validated in Polish [18]. Assessment with the KOOS scale was performed in patients undergoing ACLR assessed in the Swedish, Danish and Norwegian registries. One recent study based on the Swedish National Knee Ligament Register reported that meniscus injury at the time of reconstruction surgery was not a predictor for clinical outcome, but it was a predictor for TF [5].

The ACLR is proved to be a successful procedure. Clinical outcomes are, however, far from optimal, with one third of patients experiencing residual knee laxity and over $60 \%$ with disturbances in knee function [7]. Our study confirmed such observations. Even though we found that the number of individuals who improved was much higher than that of those who deteriorated following ACLR in the $\mathrm{KOOS}_{4}$ and in the subscales Sports and Recreation and QoL, it was only slightly higher in the KOOS subscales Pain, Symptoms and $\mathrm{ADL}$ in both groups. As expected, the surgery has a substantially smaller impact in the treatment of pain and symptoms and barely improves daily 
living activities. Our results were comparable to those reported by Barenius et al. [5] and Frobell et al. [19], who also used the KOOS scale in their studies. Notably, we used restrictive criteria with a cut-off of 10 points to detect clinically important change [21, 22], which may reduce the amount of "changers" as compared to other studies.

The analysis we made at the individual level showed that $15 \%$ of the subjects who had no concomitant meniscus surgery fulfilled severe criteria of FR. There were twice as many subjects in whom a TF was observed. The number of individuals with partial meniscal resection who fulfilled the criteria for both FR and TF was relatively low, but no conclusion should be drawn from that due to the small amount of subjects in this group.

Since there were no published reference population data in Poland, we based our analysis on the Swedish reference population [20]. The FR was defined as the lower threshold for the $95 \% \mathrm{Cl}$ of 18-34-year-old males [20], representing the most common age group and gender of subjects undergoing ACLR. The concept was first used by Barenius et al. [5], who assessed data from the Swedish National Knee Ligament Register. The percentage of subjects with both FR and TF in our series and the Swedish Register is similar [5, 31].

It has previously been reported that subjects undergoing partial meniscectomy at the time of ACLR were significantly more likely to develop radiographic knee osteoarthritis (OA) than those who had ACLR alone [23]. Subjects assessed in our study, who are at a minimum follow-up of one year, are either still in recovery or have recently restored knee function [32], but they have not yet developed clinical or radiological signs of OA. Continued observation is thus needed to assess the longer-term effects of ACLR, including development and progression of OA.

As compared to data from the Scandinavian registries, there were fewer women in our group. This reflects, however, the smaller number of women participating in sport and, consequently, lower incidence of ACL injury in females in Poland.

A potential strength of our study is that all patients were operated on by the same team and underwent an identical rehabilitation regimen. The weakest point of the present study is the relatively small sample size and only $52 \%$ power of the statistical analysis. The other limitation is that we did not measure the activity level of the patients and thus were unable to assess how many of them returned to their pre-injury level of performance. In this study, we carried out a medium-term assessment with the time from surgery to evaluation ranging from one to over 3 years. Since the clinical outcome following ACLR varies over time, the difference in assessment time can produce a bias affecting the final results. The study is, however, ongoing, and we hope it will secure better opportunities for further analyses.

In conclusion, our data suggest that patients undergoing ACLR with partial meniscectomy have a similar medium-term outcome compared to individuals with $\mathrm{ACL}$ tear alone. These data should be, however, interpreted with caution due to the small sample size. Nevertheless, even if our findings were confirmed in a larger study sample, we do not think that the current treatment strategy would be questioned. Young active subjects with traumatic meniscal tears are assigned to either meniscopexy or, if suturing is not indicated, meniscectomy, regardless of whether they undergo ACLR.

\section{Acknowledgments}

The work was supported by grant no. 5038145-3 from the Medical University of Lodz, Poland.

\section{References}

1. Frobell RB, Lohmander LS, Roos HP. Acute rotational trauma to the knee: poor agreement between clinical assessment and magnetic resonance imaging findings. Scand J Med Sci Sports 2007; 17: 109-14.

2. Gianotti SM, Marshall SW, Hume PA, Bunt L. Incidence of anterior cruciate ligament injury and other knee ligament injuries: a national population-based study. J Sci Med Sport 2009; 12: 622-7.

3. Noyes FR, Barber-Westin SD. Treatment of meniscus tears during anterior cruciate ligament reconstruction. Arthroscopy 2012; 28: 123-30.

4. Meunier A, Odensten M, Good L. Long-term results after primary repair or non-surgical treatment of anterior cruciate ligament rupture: a randomized study with a 15year follow-up. Scand J Med Sci Sports 2007; 17: 230-7.

5. Barenius B, Forssblad M, Engström B, Eriksson K. Functional recovery after anterior cruciate ligament reconstruction, a study of health-related quality of life based on the Swedish National Knee Ligament Register. Knee Surg Sports Traumatol Arthrosc 2013; 21: 914-27.

6. Spindler KP, Wright RW. Clinical practice. Anterior cruciate ligament tear. N Engl J Med 2008; 359: 2135-42.

7. Biau DJ, Tournoux C, Katsahian S, Schranz P, Nizard R. $A C L$ reconstruction: a meta-analysis of functional scores. Clin Orthop Relat Res 2007; 458: 180-7.

8. Järvelä $T$, Kannus $P$, Järvinen $M$. Anterior cruciate ligament reconstruction in patients with or without accompanying injuries: a re-examination of subjects 5 to 9 years after reconstruction. Arthroscopy 2001; 17 : 818-25.

9. Kowalchuk DA, Harner CD, Fu FH, Irrgang JJ. Prediction of patient-reported outcome after single-bundle anterior cruciate ligament reconstruction. Arthroscopy 2009; 25: 457-63.

10. Kartus JT, Russell VJ, Salmon LJ, et al. Concomitant partial meniscectomy worsens outcome after arthroscopic anterior cruciate ligament reconstruction. Acta Orthop Scand 2002; 73: 179-85.

11. Shelbourne KD, Gray T. Results of anterior cruciate ligament reconstruction based on meniscus and ar- 
ticular cartilage status at the time of surgery. Five- to fifteen-year evaluations. Am J Sports Med 2000; 28: 446-52.

12. Salmon LJ, Russell VJ, Refshauge K, et al. Long-term outcome of endoscopic anterior cruciate ligament reconstruction with patellar tendon autograft: minimum 13year review. Am J Sports Med 2006; 34: 721-32.

13. Brophy RH, Gill CS, Lyman S, Barnes RP, Rodeo SA, Warren RF. Effect of anterior cruciate ligament reconstruction and meniscectomy on length of career in National Football League athletes: a case control study. Am J Sports Med 2009; 37: 2102-7.

14. Draper V, Ladd C. Subjective evaluation of function following moderately accelerated rehabilitation of anterior cruciate ligament reconstructed knees. J Athl Train 1993; 28: 38-41.

15. Roos EM, Roos HP, Lohmander LS, Ekdahl C, Beynnon BD. Knee injury and osteoarthritis outcome score (KOOS) development of a self-administered outcome measure. J Orthop Sports Phys Ther 1998; 28: 88-96.

16. Roos EM, Roos HP, Ekdahl C, Lohmander LS. Knee injury and Osteoarthritis Outcome Score (KOOS) - validation of a Swedish version. Scand J Med Sci Sports 1998; 8: 439-48.

17. Roos EM, Lohmander LS. The Knee injury and Osteoarthritis Outcome Score (KOOS): from joint injury to osteoarthritis. Health Qual Life Outcomes 2003; 1: 64

18. Paradowski PT, Witoński D, Kęska R, Roos EM. Cross-cultural adaptation and validation of the Polish version of the Knee injury and Osteoarthritis Outcome Score (KOOS) following anterior cruciate ligament reconstruction. Health Qual Life Outcomes 2013; 11: 107.

19. Frobell RB, Roos EM, Roos HP, Ranstam J, Lohmander LS A randomized trial of treatment for acute anterior cruciate ligament tears. N Engl J Med 2010; 363: 331-42.

20. Paradowski PT, Bergman S, Sunden-Lundius A, Lohmander LS, Roos E. Knee complaints vary with age and gender in the adult population. Population-based reference data for the Knee injury and Osteoarthritis Outcome Score (KOOS). BMC Musculoskelet Disord 2006; 7: 38.

21. Ehrich EW, Davies GM, Watson DJ, Bolognese JA, Seidenberg BC, Bellamy N. Minimal perceptible clinical improvement with the Western Ontario and McMaster Universities osteoarthritis index questionnaire and global assessments in patients with osteoarthritis. J Rheumatol 2000; 27: 2635-41.

22. Paradowski PT, Englund $M$, Roos EM, Lohmander LS. Similar group mean scores, but large individual variations, in patient-relevant outcomes over 2 years in meniscectomized subjects with and without radiographic knee osteoarthritis. Health Qual Life Outcomes 2004; 2: 38 .

23. Magnussen RA, Mansour AA, Carey JL, Spindler KP. Meniscus status at anterior cruciate ligament reconstruction associated with radiographic signs of osteoarthritis at 5- to 10-year follow-up: a systematic review. J Knee Surg 2009; 22: 347-57.

24. Wu WH, Hackett T, Richmond JC. Effects of meniscal and articular surface status on knee stability, function, and symptoms after anterior cruciate ligament reconstruction: a long-term prospective study. Am J Sports Med 2002; 30: 845-50

25. Tow BP, Chang PC, Mitra AK, Tay BK, Wong MC. Comparing 2-year outcomes of anterior cruciate ligament reconstruction using either patella-tendon or semiten- dinosus-tendon autografts: a non-randomised prospec tive study. J Orthop Surg (Hong Kong) 2005; 13: 139-46.

26. Tandogan RN, Taşer O, Kayaalp A, et al. Analysis of me niscal and chondral lesions accompanying anterior cruciate ligament tears: relationship with age, time from injury, and level of sport. Knee Surg Sports Traumato Arthrosc 2004; 12: 262-70.

27. Joseph C, Pathak SS, Aravinda M, Rajan D. Is ACL reconstruction only for athletes? A study of the incidence of meniscal and cartilage injuries in an ACL-deficient athlete and non-athlete population: an Indian experience. Int Orthop 2008; 32: 57-61.

28. Yoo JC, Ahn JH, Lee SH, Yoon YC. Increasing incidence of medial meniscal tears in nonoperatively treated anterior cruciate ligament insufficiency patients documented by serial magnetic resonance imaging studies. Am J Sports Med 2009; 37: 1478-83.

29. Lind M, Menhert F, Pedersen AB. The first results from the Danish $A C L$ reconstruction registry: epidemiologic and 2 year follow-up results from 5,818 knee ligament reconstructions. Knee Surg Sports Traumatol Arthrosc 2009; 17: 117-24.

30. Hambly K, Griva K. IKDC or KOOS. Which one captures symptoms and disabilities most important to patients who have undergone initial anterior cruciate ligament reconstruction? Am J Sports Med 2010; 38: 1395-404.

31. Granan LP, Forssblad M, Lind M, Engebretsen L. The Scandinavian ACL registries 2004-2007: baseline epidemiology. Acta Orthop 2009; 80: 563-7.

32. Hill GN, O'Leary ST. Anterior cruciate ligament reconstruction: the short-term recovery using the Knee Injury and Osteoarthritis Outcome Score (KOOS). Knee Surg Sports Traumatol Arthrosc 2013; 21: 1889-94 